\title{
Post-traumatic digital flexion contracture (checkrein deformity)
}

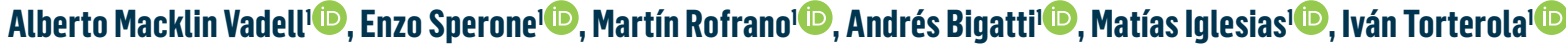 \\ 1. Sanatorio Finochietto, Ciudad Autónoma de Buenos Aires, Buenos Aires, Argentina.
}

\begin{abstract}
Objective: The aim of this study is to present a series of 8 patients, describing their clinical picture and assessing their treatment using plantar approach.

Methods: We retrospectively assessed 8 patients, all of which had a history of trauma. The clinical characteristics of these cases and postoperative results were investigated.

Results: Seven men and 1 woman with a mean age of 29 years were included. The follow-up period ranged from 6 to 28 months (mean 22 months). The deformity manifested from 5 to 24 months after the triggering injuries (mean 9.8 months). The hallux was the only digit affected in 1 patient, while the others presented with involvement of 1 or more small toes. There were no postoperative complications, and patients showed to be satisfied with functional outcomes.
\end{abstract}

Conclusion: Post-traumatic digital flexion contracture is an infrequent disease of unknown etiology. Lengthening of the flexor hallucis longus using a plantar approach, whether at the level of the midfoot or the toe, represents an alternative with satisfactory outcomes.

Level of Evidence IV; Therapeutic Study; Case Series.

Keywords: Contracture; Hallux; Foot deformities, acquired.

\section{Introduction}

Flexion contracture at the hallux interphalangeal joint that presents after trauma is known as checkrein deformity and can manifest, to a lesser extent, with extension contracture of the metatarsophalangeal joint and also include small toes. This contracture is flexible and is produced during ankle dorsiflexion and is totally or partially corrected during plantar flexion (Figure 1). Furthermore, it occurs due to traction by the flexor hallucis longus ( $F H L$ ) and, although its cause is not clear, it is commonly described as tendon entrapment at the level of the ankle or the hindfoot ${ }^{(1)}$. In the literature, checkrein deformity has been associated with ankle fractures, whether surgically treated or not, as well as with leg, pilon, talar, and calcaneal fractures ${ }^{(2-6)}$. The FHL may be entrapped in the fibrous scar tissue or in the bony callus ${ }^{(2-7)}$. Some authors consider that FHL shortening is caused by the development of subclinical deep compartment syndrome ${ }^{(6)}$. Checkrein deformity usually appears spontaneously from 2 to 12 months after the triggering injury ${ }^{(4,8)}$.
Clawson $^{(9)}$, in 1974, was the first to describe checkrein deformity as a dynamic process associated with lower limb fractures.

Since then, several approaches have been described for its treatment, which consists of lengthening of the FHL tendon ${ }^{(1-4,6-8)}$. If necessary, release of flexor digitorum communis (FDC) tendon or distal tenotomy is performed ${ }^{(6)}$. Endoscopic release of the fibrous tissue that entraps the $\mathrm{FHL}$ tendon with no need for its lengthening has also been described(10).

The aim of this study is to present a series of 8 patients with checkrein deformity, describing their clinical picture and assessing their treatment using a plantar approach.

\section{Methods}

This study was approved by the Ethics Committee of our institution.

We retrospectively evaluated 8 patients that had been operated by the same surgical team from 2011 to 2019. Four of
Study performed at the Sanatorio Finochietto, Ciudad Autónoma de Buenos Aires, Buenos Aires, Argentina.

Correspondence: Andrés Bigatti. Arcos $27281^{\circ} 14$, Ciudad Autónoma de Buenos Aires - 1428, Buenos Aires, Argentina. E-mail: andres_bigatti9@hotmail.com Conflicts of interest: none. Source of funding: none. Date received: April 11, 2021. Date accepted: July 02, 2021. Online: August 31, 2021.
How to cite this article: Macklin Vadell A

Sperone E, Rofrano M, Bigatti A, Iglesias

M, Torterola I. Post-traumatic digital flexion

contracture (checkrein deformity). J Foot Ankle. 2021;15(2):146-9. 
these patients initially presented with closed leg fracture and were treated with intramedullary nailing; the remaining cases were the following: a closed leg fracture treated with casting, a closed fracture of the distal tibia treated with an external tutor, a closed fracture-dislocation of the ankle, and a closed ankle fracture, the last 2 treated with open reduction and internal fixation (Table 1).

The main patients' reasons for consultation were pain on the tip of the hallux, difficulty in wearing closed shoes, and gait changes. On physical examination, all patients presented with dynamic deformity of the hallux. Flexion of the interphalangeal joint and extension of the metatarsophalangeal joint were observed at ankle dorsiflexion, but this deformity was reduced at ankle plantar flexion. Weight bearing frontal and profile $\mathrm{X}$-rays of leg, ankle and foot were requested for all patients, as well as echography and nuclear magnetic resonance (NMR), for diagnostic purposes.

Furthermore, the present study assessed the time elapsed from the initial injury to the onset of the deformity and whether there was involvement of small toes.

Patients were surgically treated with Z-lengthening of the FHL tendon and release of FDC when necessary. Seven of these patients were operated using a plantar approach, and
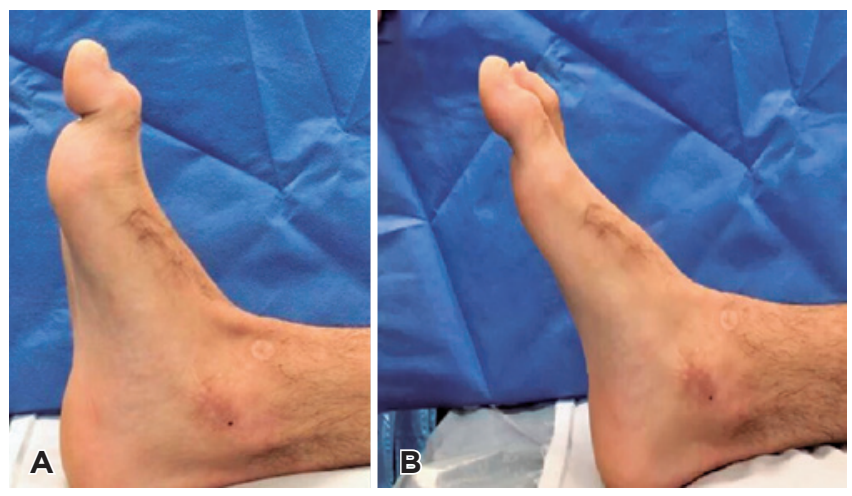

Figure 1. A) Flexion of the interphalangeal joint of the hallux at ankle dorsiflexion. B) Correction of the deformity at ankle plantar flexion. only 1 case was approached distally at the level of the hallux. Below we describe the surgical technique employed in each approach.

The 8 operated patients were clinically assessed for pain according to the visual analog scale (VAS), type of shoe (possibility of using any type of shoes without making any change to them, limitation in the use of certain types of shoes, need for custom-made shoes), and degree of satisfaction (whether the patient would undergo surgery again).

\section{Surgical technique and postoperative care: Plantar midfoot approach}

The patient was placed in a supine position on the operating table, surgical fields were positioned according to the technique; moreover, a hemostatic cuff was used on the homolateral thigh, with enhancement at the level of the contralateral buttock. A longitudinal incision of approximately $3 \mathrm{~cm}$ was made, lateral to the abductor muscle of the hallux and $2 \mathrm{~cm}$ proximal to the medial sesamoid bone. Tissue dissection was performed in layers, and FHL and FDC tendons were identified. Z-plasty lengthening was performed in order to achieve complete extension of the interphalangeal and metatarsophalangeal joints of the hallux with the ankle in the neutral position and suture with a non-resorbable monofilament thread (Figure 2). If there was involvement of small toes, tendinous connections were divided between the $\mathrm{FHL}$ and the FDC. If deformity persisted, percutaneous tenotomy was performed at the proximal level of the distal interphalangeal joint of each toe. Layered closure was performed. In the postoperative period, immobilization is maintained with a short cast boot including the hallux and the ankle at $90^{\circ}$ for 6 weeks. Subsequently, the cast was removed, and weight bearing was initiated with a Walker boot and progressive mobilization of ankle and hallux.

\section{Digital approach}

The patient was placed in a supine position on the operating table, surgical fields were positioned according to the technique, a hemostatic cuff was placed on the homolateral thigh, with enhancement at the level of the contralateral

Table 1. Analysis of patients' characteristics.

\begin{tabular}{|c|c|c|c|c|c|c|c|}
\hline & Age & Sex & Initial trauma & Initial treatment & Time of onset & Small toes & Approach \\
\hline 1 & 37 & M & Leg Fx & IMN & 14 months & All & Midfoot \\
\hline 2 & 26 & M & Distal tibial Fx & External tutor & 6 months & None & Midfoot \\
\hline 3 & 21 & M & Ankle FD & ORIF & 24 months & $2^{\text {nd }}$ & Midfoot \\
\hline 4 & 43 & $\mathrm{~F}$ & Leg Fx & IMN & 8 months & $2^{\text {nd }}$ and $3^{\text {rd }}$ & Midfoot \\
\hline 5 & 13 & M & Leg Fx & Casting & 5 months & $2^{\text {nd }}$ & Midfoot \\
\hline 6 & 29 & M & Leg Fx & IMN & 7 months & $2^{\text {nd }}$ & Midfoot \\
\hline 7 & 27 & $M$ & Ankle Fx & ORIF & 9 months & $2^{\text {nd }}$ & Midfoot \\
\hline 8 & 39 & M & Leg Fx & IMN & 6 months & $2^{\text {nd }}$ & Digital \\
\hline
\end{tabular}

*M (Male); F: (Female); Fx: (Fracture); FD: (Fracture-Dislocation); IMN: (Intramedullary Nailing); ORIF: (Open Reduction and Internal Fixation). 
buttock. A $3-\mathrm{cm}$ incision is made on the plantar surface of the hallux. Tissue dissection was performed in layers, and FHL was identified. Z-plasty lengthening was performed in order to achieve complete extension of the interphalangeal and metatarsophalangeal joints of the hallux with the ankle in the neutral position and suture with a non-resorbable monofilament thread (Figure 3 ). In case of involvement of small toes, percutaneous tenotomy was performed at the proximal level of the distal interphalangeal joint of each toe. Layered closure was performed. In the postoperative period, immobilization was maintained with a short cast boot including the hallux and the ankle at $90^{\circ}$ for 6 weeks. Subsequently, the cast was removed, and weight bearing was initiated with a Walker boot and progressive mobilization of ankle and hallux.
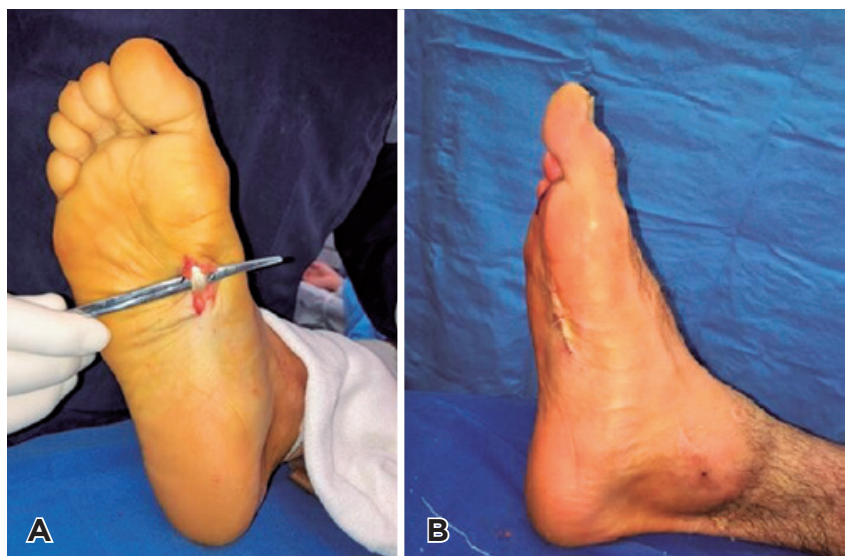

Figure 2. A) Identification of the FHL tendon using a plantar midfoot approach. B) Correction of the deformity.

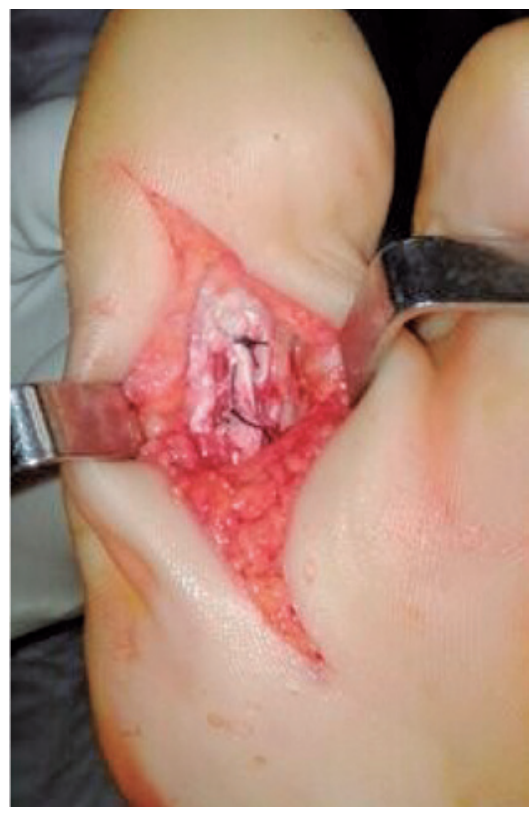

Figure 3. Digital approach.

\section{Results}

Eight patients were retrospectively assessed, 7 men and 1 woman, with a mean age of 29 years (from 13 to 43 years). The postoperative follow-up of the 8 treated patients lasted for a minimum of 6 and a maximum of 28 months (mean 22 months).

Checkrein deformity manifested from 5 to 24 months after the initial injury (mean 9.8 months) (Table 1). This deformity affected only the hallux in 1 patient (12.5\%); conversely, it also affected the second toe in 5 patients (62.5\%), the second and the third toes in 1 patient (12.5\%), and all small toes in 1 patient (12.5\%).

The patients did not have neither wound complications nor postoperative neurovascular injuries. There were no recurrences nor need for reintervention. Seven patients reported absence of pain, and 1 reported mild pain. The 8 patients answered that they could wear any type of shoes without making any change to them and that they would undergo surgery again.

There were no evident functional differences between the cases in which lengthening of the FHL tendon was performed at the level of midfoot and the case that was approached at the level of the hallux.

\section{Discussion}

Post-traumatic digital flexion contracture, known as checkrein deformity, consists of the dynamic flexion of the interphalangeal joint and, to a lesser extent, extension of the metatarsophalangeal joint of the hallux. It manifests at ankle dorsiflexion and is totally or partially corrected at ankle plantar flexion. Small toes may also be affected, due to the connections between FHL and FDC tendons.

Checkrein deformity has been mainly related to leg and ankle fractures in the literature, which is consistent with our series. However, the cause of this deformity has not been elucidated yet. There have reports of adherence of the FHL tendon to the scar tissue surrounding the fracture ${ }^{(7)}$ or entrapment of this tendon in the callus formed after fracture union $^{(2)}$. However, none of these 2 situations could explain the development of this deformity in patients who do not have a fracture ${ }^{(8)}$. Feeney et al. ${ }^{(6)}$ consider subclinical compartment syndrome of the leg as a possible explanation in those cases. The distal portion of the FHL muscle is found in a smaller and deeper compartment; thus, a localized increase in pressure could generate ischemia and muscle retraction(11).

NMR and echography allow us to visualize the muscle injury and the relationship of nervous and vascular structures with possible areas of fibrosis, edema, and hematoma, but they did not define the specific cause of the problem.

Clinically, the patients may present pain on the tip of the hallux due to hyperpressure, difficulty in crouching, gain limitation, and impossibility to wear certain type of shoes, especially women. 
Kinesic treatment is not beneficial for these patients. We consider that treatment is surgical and consists of lengthening of the FHL tendon.

Feeney et al. ${ }^{(6)}$ reported goods outcomes with Z-plasty through an internal retromalleolar approach in 10 patients. Lee et al. ${ }^{\left({ }^{8}\right)}$ found 2 partial recurrences and 1 complete recurrence in 5 patients using the same approach.

Lee et al.(1) reported good outcomes and no case of recurrence with an approach at the level of the tarsal tunnel in 8 patients. They did not have neurovascular complications; however, the authors describe the possibility of developing tarsal tunnel syndrome, thus highlighting the importance of taking care not to damage the neurovascular bundle.

Tenotomy and interphalangeal arthrodesis of the hallux may be good options, especially in the case of recurrence ${ }^{(12)}$.

In our series, we observed very good outcomes with Z-lengthening of the $\mathrm{FHL}$ through plantar midfoot approach in 7 patients and with digital approach in 1 patient. There were no recurrences, and no differences were observed in final outcomes between the 2 approaches. Other authors have published good outcomes with the plantar midfoot approach $^{(8,13)}$. Conversely, there is no clear evidence on the outcomes with distal approach. The need of performing distal tenotomies in case of involvement of small toes may be a disadvantage of the digital approach. Finally, the possibility of neurovascular injuries and postoperative adherences are lower with these 2 approaches compared to those using the tarsal tunnel and the internal retromalleolar area ${ }^{(1,8)}$.

\section{Conclusion}

Post-traumatic digital flexion contracture is an infrequent injury of unclear etiology. It may be associated with different conditions, the most common of which being leg and ankle fractures. Plantar approaches, at the level of the midfoot and the toe, allow to lengthen the FHL tendon enough to correct the deformity with low risk of neuromuscular injuries and postoperative adhesions.

Authors' contributions: Each author contributed individually and significantly to the development of this article: AMV *(https://orcid.org/OOOO-OOO20384-4044) Conceived and planned the activities to execute the work, participated in the interpretation of the results, performed the surgeries and approved the final versión; ES *(https://orcid.org/0000-0001-5028-9584) Conceived and planned the activities to execute the work, participated in the interpretation of the results, performed the surgeries; MR *(https://orcid.org/0000-0003-1947-8218) Participated in the interpretation of the results and performed the surgeries; AB *(https://orcid.org/0000-0003-1690-025X) participated in the bibliographic search and executing the work; MI *(https://orcid. org/0000-0002-6336-6080) Participated in data collection and clinical evaluation of patients; IT *(https://orcid.org/0000-0001-9210-9051) Participated in the bibliographic search and the clinical evaluation of the patients. All authors read and approved the final manuscript. *ORCID (Open Researcher and Contributor ID) (iD).

\section{References}

1. Lee JH, Kim YJ, Baek JH, Kim DH. Z-plasty of the flexor hallucis longus tendon at tarsal tunnel for checkrein deformity. J Orthop Surg (Hong Kong). 2016;24(3):354-7.

2. Kim SH, Lee KT, Smith RW, Park YU. Checkrein deformity secondary to entrapment of FHL after talus fracture: a case report. Foot Ankle Int. 2010;31(4):336-8.

3. Rosenberg GA, Sferra JJ. Checkrein deformity--an unusual complication associated with a closed Salter-Harris Type II ankle fracture: a case report. Foot Ankle Int. 1999;20(9):591-4.

4. Sanhudo JA, Lompa PA. Checkrein deformity--flexor hallucis tethering: two case reports. Foot Ankle Int. 2002;23(9):799-800.

5. Carr JB. Complications of calcaneus fractures entrapment of the flexor hallucis longus: report of two cases. J Orthop Trauma. 1990;4(2):166-8.

6. Feeney MS, Williams RL, Stephens MM. Selective lengthening of the proximal flexor tendon in the management of acquired claw toes. J Bone Joint Surg Br. 2001;83(3):335-8.

7. Leitschuh PH, Zimmerman JP, Uhorchak JM, Arciero RA, Bowser L. Hallux flexion deformity secondary to entrapment of the flexor hallucis longus tendon after fibular fracture. Foot Ankle Int. 1995; 16(4):232-5

8. Lee HS, Kim JS, Park SS, Lee DH, Park JM, Wapner KL. Treatment of checkrein deformity of the hallux. J Bone Joint Surg Br. 2008; 90(8):1055-8.

9. Clawson DK. Claw toes following tibial fracture. Clin Orthop Relat Res. 1974;(103):47-8.

10. Lui TH. Endoscopic adhesiolysis of the flexor hallucis longus muscle. Foot Ankle Spec. 2014;7(6):492-4.

11. Sassu P, Acland RD, Salgado CJ, Mardini S, Ozyurekoglu T. Anatomy and vascularization of the flexor hallucis longus muscle and its implication in free fibula flap transfer: an anatomical study. Ann Plast Surg. 2010;64(2):233-7

12. Holcomb TM, Temple EW, Barp EA, Smith HL. Surgical correction of checkrein deformity after malunited distal tibia fracture: a case report. J Foot Ankle Surg. 2014;53(5):631-4.

13. Miranda BR, Barroco RS, Nishikawa DRC, Oliveira LZP, Ghani MBA Checkrein deformity treated through a forefoot approach: a case report. Sci J Foot Ankle. 2019;13(4):259-63. 